Théologiques

Théologiques

\title{
Heureux comme Dieu en exégèse?
}

\section{Olivette Genest}

Volume 6, numéro 2, octobre 1998

Dieu interdit

URI : https://id.erudit.org/iderudit/024962ar

DOI : https://doi.org/10.7202/024962ar

Aller au sommaire du numéro

\section{Éditeur(s)}

Faculté de théologie de l'Université de Montréal

\section{ISSN}

1188-7109 (imprimé)

1492-1413 (numérique)

Découvrir la revue

\section{Citer cet article}

Genest, O. (1998). Heureux comme Dieu en exégèse? Théologiques, 6(2), 51-59. https://doi.org/10.7202/024962ar

\section{Résumé de l'article}

Occupée d'écrits que Dieu remplit marge-à-marge, Pexégèse biblique parle-t-elle forcément de lui? L'ambiguïté factuelle de la réponse suscite cette question insolite. L'auteure distingue entre omniprésence, absence et présence de Dieu en exégèse; entre Dieu de la Bible, Dieu de l'exégèse et Dieu familier des croyants; entre exégèse et grâce. Elle souhaite un rajustement de la démarche exégétique qui, par-delà son impressionnante activité heuristique, revienne à la respiration du texte et lui laisse le soin de parler de Dieu, si et quand l'acte de lecture le requiert. Cependant, le Dieu de la Bible, éclairé par le Dieu de l'exégèse, peut-il entrer de plain-pied dans notre époque et pouvons-nous, sans plus, le rejoindre sans quitter la nôtre? Il appartient aussi à l'exégèse d'oeuvrer ici au service de la lecture. 
Théologiques 6/2 (1998) 51-59.

\title{
Heureux comme Dieu en exégèse?
}

\author{
Olivette GENEST \\ Faculté de théologie \\ Université de Montréal
}

Dieu est-il heureux en exégèse, comme il l'est en France, paraîtil, d'après l'expression allemande bien connue? Ou y est-il interdit de séjour comme dans tant d'autres sciences humaines? Car, l'interprétation des Écritures, des Saintes Écritures, naguère science divine est maintenant devenue science humaine. L'organigramme des uni versités n'est pas le seul responsable de cette mutation mais aussi une évolution interne qui a cherché à la dégager des positions confessionnelles qui lui servaient d'instruments de lecture.

Depuis la brèche opérée par le Tractatus theologico-politicus de Spinoza en 1670, l'introduction de la raison critique dans son champ du savoir a donné naissance à une panoplie de méthodes adaptées à son objet. Avec les exigences de la formation qu'elle requiert, les rigueurs de son exercice technique et ses longs laboratoires sur textes, elle tient, pour une part, des sciences exactes. Victime de son statut scientifique, elle s'est retrouvée cantonnée dans l'heuristique, par des facteurs et externes et internes encore une fois. Les théologiens se réservaient d'office l'opération herméneutique et la pratique des exégètes émergeait difficilement du nécessaire éclairage de la distance historique du corpus biblique. Le symptôme aigu de ce malaise a éclaté dans la création de la sous-discipline nommée " théologie biblique ». Dans une ambiguïté méthodologique non encore résolue, son existence traduit chez les exégètes la réclamation du droit à l'interprétation.

Toute marquée qu'elle est d'un net profil scientifique, l'exégèse concerne également le monde de la croyance, de la foi et de la spiritualité. Elle a pour objet le texte considéré comme sacré, avec ou sans sa clôture canonique. Sous cet angle, Dieu le remplit, à titre d'auteur et de Parole, comme il sature la face scientifique de son 
activité en tant que personnage principal. En exégèse, Dieu est partout. Il est le texte, tout le texte. "Dieu est partout, pourquoi ne le voyons-nous pas? », s'interrogeait le Petit Catéchisme romain de préparation à la première communion. En le nommant constamment, l'exégèse parle-t-elle de lui? Et, si elle en parle, de qui parle-t-elle dans la langue de l'enseignement et de l'écriture?

\section{L'omniprésence et l'absence de Dieu en exégèse}

\subsection{L'exégèse parle-t-elle de Dieu?}

L'exégèse parle beaucoup, et savamment, d'une multitude de sujets. Dieu y a une grande rivale : la tâche descriptive d'un texte très ancien, terre d'abondance pour l'heuristique. L'exégèse ne dit encore rien de Dieu dans son attention inépuisable à l'étude des manuscrits bibliques, à l'analyse de la genèse du texte et de ses sources composites, aux problèmes philologiques de l'archéologie des mots et des pierres. Tout travail utile, incontournable, mais œuvrant dans l'antichambre de l'interprétation. Quelle chance pour Dieu que la redécouverte de la dimension synchronique des écrits!

Il n'y habite pas seul cependant et on peut constater l'impact des approches et des méthodes adoptées sur son image même. Ainsi certains commentaires récents ne lui concèdent qu'une ombre évanescente dans des passages extraits des épîtres pauliniennes à des fins christologiques, alors que le contexte lui attribue le rôle de sujet grammatical, sémiotique et sémantique, alors que le Christ n'y est discursivisé que comme "Fils de". Impact sur son image également que d'aborder la Bible avec le projet d'y trouver le ou des traits modernes qui correspondraient à notre image à nous. C'est souvent de nous que nous parlons dans ces tentatives.

Au cours d'exégèse, la bigarrure des auditoires, riche en ellemême, ajoute néanmoins à la difficulté de discourir sur Dieu, dans le respect dû aux nombreux « dieux " apportés en classe par étudiants et étudiantes. Auditoires multiethniques, multiconfessionnels, illus trant tout ce que la religion peut véhiculer de meilleur et de pire : superstitions, conversions à certitudes parfois bruyantes, dépouille. ments de la maturité, étiquette "moderne " avec ou sans connais sance de la critique de la modernité, recours commode au mystère devant le spectre de la déconstruction, manifestations d'une transpa- 
rence et d'une pureté qu'on croirait facilement égarée dans cette fin de siècle et de millénaire. Difficulté surtout de s'adresser à deux types de personnes : les croyants et les non-croyants, quelques fois les incroyants; complexité de l'identité du professeur entre son évolution personnelle et sa formulation disciplinaire, à commencer par la notion de foi. Embarras enfin entre l'information scientifique à transmettre et les attentes diversifiées de l'auditoire, entre formation à la recherche d'une minorité et préparation à l'intervention à buts variés du plus grand nombre.

Dans ce dédale, peu de couloirs résonnent du nom de Dieu. Affairés qu'ils sont à rencontrer des exigences académiques, à poursuivre tel sillon de recherche, à meubler telle case de leur curriculum vitoe, je ne crois pas, de mon angle de vision, que les exégètes consacrent beaucoup de temps, quantitatif et qualitatif, à parler de Dieu. À la bibliothèque, les traités d'exégèse laissent sereinement à leurs usagers le soin de combler la distance entre ce qu'ils offrent et la quête qui les y a conduits. Leur faudrait-il passer au rayon, plus savoureux, de la spiritualité?

\subsection{L'exégèse doit-elle parler de Dieu?}

Dans ses publications et la transmission de ses résultats et travaux en cours, l'exégèse n'est pas, de sa nature, guide de méditation, de retraite, d'animation spirituelle, de catéchèse, de préparation à l'homélie sur des passages qu'elle connaît mieux que personne. Ces différentes activités sur le texte biblique relèvent d'arts et de techniques particulières à chacune. L'homélie, par exemple, n'est pas le lieu d'un cours d'exégèse, et ce dernier ne doit pas tourner non plus à l'homélie prolongée.

On attend de l'exégèse qu'elle informe et forme à l'esprit cri tique. Elle n'a pas à prêcher Dieu, est-ce à dire qu'occupée d'un livre sur Dieu, dit de Dieu et plein de Dieu, on ait à la louer d'être aussi discrète à son égard? On peut souhaiter que, sans confusion de perspectives, elle laisse au texte la liberté de parler de Dieu. Qu'elle enseigne à le lire et elle aura rempli sa mission propre. Ce qui suppose qu'elle s'attache davantage au niveau des significations qu'il produit, appelées communément sens.

Les découpages méticuleux qui l'ont occupée au vingtième siècle ont été fort utiles à la progression des connaissances et même à 
l'épuration de la croyance. Nous les avons tous admirés, mais n'y a-ton pas souvent perdu de vue l'horizon du texte? Trop d'études s'attardent encore dans une philologie positiviste ou adoptent comme base de départ les statistiques des concordances. Par ailleurs si commode, la répartition en versets a brisé l'enchaînement du texte. Il n'est pas étonnant que, dans l'habitude généralisée du démembrement systématique, les classiques de la Théologie biblique ait eu tant de mal à prendre pied et à se fonder méthodologiquement, malgré leurs richesses certaines. Dans un texte traité selon les lois de la nature d'un texte, les bribes de Dieu, mesurées par le hublot des versets, s'éclaireraient d'elles-mêmes, non par collage mais par retour à la respiration d'un corpus qui ne peut pas ne pas parler de lui.

Quel Dieu, à la fois familier et étranger, pouvons-nous attendre de ce rajustement de la démarche exégétique? Surtout, n'appar tiendrait-il pas à l'exégèse de discuter de ses rapports à notre temps? Peut-il entrer de plain-pied dans notre époque, sans modification d'aucun de ses traits; pouvons-nous le rejoindre dans la sienne sans abandon d'aucun des nôtres?

\subsection{De quel Dieu « exégétique "s'agit-il?}

L'exégèse reconnaît au Dieu de la Bible des traits sémitiques, hébraïques, gréco-romains et chrétiens. Une galerie de ses portraits au fil des livres et des époques accuse des différences entre le Dieu des Juges en Israël, l'Abba de Jésus-Christ et celui des néo-chrétiens venus de la gentilité. Faudrait-il identifier un Dieu par Testament ou un Dieu pour les deux dans l'optique de la Bible chrétienne où l'Ancien Testament trouve son aboutissement dans le Nouveau? Ou surimposer une image testamentaire sur l'autre et en déduire une troisième qui serait celle, combinée, du Dieu des chrétiens? Ou, à l'inverse, dédoubler les images, "déshelléniser " le Dieu de la théologie et des églises pour le "re-bibliser "?

La remontée vers les sources textuelles composites, vers la source la plus ancienne a longtemps captivé l'activité heuristique de l'exégèse. Elle a souvent oublié que, si la source première éclaire certains aspects du texte, elle est elle-même remaniée par le dernier niveau de rédaction. De façon générale, le retour aux sources, à la frâicheur des re-découvertes, comporte une forte dose d'ambiguïté, même au nom du retour à la simplicité pratiqué par le postmodernisme le plus 
décapant ${ }^{1}$. À côté du Dieu impassible de la philosophie grecque, le Dieu vulnérable de la Bible, atteint par la souffrance jusque dans sa gloire suprême, exerce une grande fascination. Le lecteur du XXe siècle doit-il pour autant revenir à un Yahvé très anthropomorphisé?

Harold Bloom, professeur d'Humanités à Yale, également professeur aux Études anglaises de l'Université de New York, s'appuyant sur la traduction de David Rosenberg, hébraissant, poète et essayiste, s'est amusé à appliquer au Yahvé de la source littéraire dite "yahviste " (J) dans le Pentateuque la méthode qu'il emploie pour l'analyse des personnages de Shakespeare. Est apparu un acteur peu édifiant, en manque aigu des retouches à son caractère que lui apporte la combinaison rédactionnelle de $\mathrm{J}$ avec les sources dites "élohiste " $(\mathrm{E})$, "sacerdotale " $(\mathrm{P})$ et « deutéronomiste " $(\mathrm{D})$, sources identifiées par les exégètes dans la version reçue de nos bibles! S'est imposée surtout la conclusion que l'assertion de continuité avec le yahvisme du judaïsme fondé au deuxième siècle était aussi arbitraire, écrivent les auteurs, que la réclamation chrétienne de la même filiation ${ }^{2}$.

Un livre ne fait pas une certitude. Le débat est ouvert sur les avatars du Dieu de la Bible, sur son histoire et son évolution. Le chris tianisme peut-il annoncer sans plus le Dieu des Hébreux bibliques? L'originalité de Jésus s'identifiant à son Père a bousculé le monothéisme juif et, après la venue du Christ, le Dieu des chrétiens s'est développé en Trinité. S'ajoute, pour l'exégèse, la nécessité de réflé chir sur ses propres habitudes d'interprétation. Qu'en est-il de la saine démythisation de certains passages de l'Ancien Testament qu'elle n'ose poursuivre quand il s'agit de l'appliquer au Nouveau Testament? La question du Dieu biblique ne peut se borner à une simple description, déjà épineuse en elle-même, à une photographie du texte titrée: Voici Dieu. Dans l'état actuel de la recherche, et

1 «La simplicité n'est pas la même chose que le passé et pourquoi la simplicité devrait-elle être un retour? Un retour à quoi, du reste? Vous voyez de la simplicité dans le passé, vous? »Intervention du compositeur américain Frédéric RZWESKI dans une discussion radiophonique sur "Qu'est-ce que le postmodernisme musical?" rapportée dans la revue Circuit de la Faculté de musique de l'Université de Montréal, volume 1, numéro 1, 1991, p. 19.

2 Voir H. BLOOM, D. ROSENBERG, The Book of J. New York, Grove Weidenfeld, 1990, p. 284. 
tout en tenant compte des avancées exploratoires actuelles de la théologie biblique, on peut constater une paradoxale juxtaposition d'omniprésence et d'absence dans le discours exégétique sur Dieu.

\section{Exégèse et présence de Dieu}

L'exégèse a pour mission l'étude des sources chrétiennes scripturaires. Elle ne peut donc pas ne pas parler de Dieu, mais on ne peut lui demander ce qu'elle ne peut donner, c'est-à-dire sa présence. Cette attente peut se trouver chez les auditeurs et lecteurs de ses travaux; il leur revient à eux de faire leur miel de ce qu'elle leur offre. À mon avis, on peut pratiquer l'exégèse sans poser son adhésion personnelle à la proposition du texte et, par un travail littéraire et historique rigoureux et consciencieux, éclairer le croyant lui-même. Cette opinion controversée n'a jamais fait l'unanimité. Les partisans opposés alignent des raisons sérieuses à l'appui de leur thèse, qui valent pour tout le domaine de la théologie ${ }^{3}$. De toute façon, l'ordre de l'étude des textes fondateurs differe de celui de la présence. De ce dernier vivent et discourent les mystiques, sans exclure qu'un ou une exégète ne puisse également se doubler d'un-e mystique.

La même distinction se retrouve entre lesdites "preuves" de l'existence de Dieu et le sentiment de sa présence. La connaissance des premières, que Thomas d'Aquin nommait plus modestement "voies pour aller à Dieu", ne réussit pas à guérir les absences

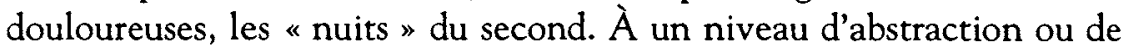
théorisation plus large encore, dans toute communication personnelle on peut différencier son contenu et la relation elle-même, la

3 Depuis les premières didascalies d'Antioche et d'Alexandrie, des propositions à valeur de théorèmes ont alimenté la dispute: il faut comprendre pour croire ou il faut croire pour comprendre; la raison rend la foi vraisemblable ou la foi exhausse la portée de la raison; dans la lecture de la Bible, la foi est une condition sine qua non de compréhension ou seul le non-croyant peut arriver à une lecture objective. Les réflexions de CULLMANN et de BULTMANN, dans la partie méthodologique de leurs Théologies du Nouveau Testament méritent attention devant cette dichotomie inextricable. Le premier avertit que l'expérience personnelle de foi peut aussi être une source d'erreur et ne pas coïncider avec celle des héros bibliques. Pour le second, la foi ne peut constituer un instrument épistémologique et méthodologique. 
seconde englobant le premier ${ }^{4}$. Le contenu, ou l'information transmise, s'étale selon une syntaxe logique très complexe et très commode. La relation, elle, se perçoit directement, induit un comportement et possède une sémantique appropriée. On le sait par expérience, certaines communications déficientes manquent ou de contenu ou de syntonie.

La leçon d'exégèse, orale et écrite, qui respecte ses objectifs, doit transmettre un maximum de richesses cognitives, à tout le moins une dose convenable d'information. Sa syntaxe propre respectée et enrichie, elle doit aussi établir le contact avec son pôle de réception. Doit-elle également ouvrir la relation avec un troisième sujet, avec le Dieu dont elle parle? Dieu est-il invité à la leçon d'exégèse?

Encore une fois, les opinions divergent. À mon avis, la communication exégétique planifiée est réussie dès qu'elle passe entre ses destinateurs-exégètes et ses destinataires auditeurs-lecteurs ${ }^{5}$. Le rapport à Dieu s'inscrit sur une autre ligne de communication, cette fois celle qui va de l'auditeur-lecteur à Dieu, à situer plutôt, pour le sujet qui nous intéresse, sur l'axe non plus de l'enseignement mais sur celui de la lecture de la Bible, entre texte et lecteur croyant. Cependant, même les théories sur la lectio divina ou lecture spirituelle varient. Pour certains, elle prépare à l'oraison, pour d'autres elle est le lieu du contact avec Dieu.

En définitive, la réponse réside au pôle de réception de l'exégèse, et non au pôle de son énonciation, soit dans la requête qu'apporte la personne qui fréquente les études exégétiques. À elle de donner la signification personnelle supplémentaire. À l'exégète de s'effacer devant la primauté du texte, même s'il passera toujours dans ses travaux quelque chose de sa personnalité, comme dans toutes les interprétations.

4 Ces considérations s'appuient sur le livre de Paul WATZLAWICK, Janet Helmick BEAVIN et Don D. JACKSON, Une logique de la communication. Paris, Seuil, 1972 (original américain de 1967), chapitre 2: Propositions pour une axiomatique de la communication, pp. 45-69.

5 Il n'y a pas lieu ici de discuter plus avant le schéma de la communication en général et l'ordre de production entre signification transmise et phénomène de la communication, entre objet et mécanisme de transmission. 
Dans l'attention élaborée accordée à un texte qui parle de lui, Dieu n'aurait donc pas d'emblée la part du roi, du lion... de Dieu? Il n'y vivrait pas aussi largement et heureusement que "Dieu en France "? Jouirait-il d'un statut plus confortable dans les autres secteurs du champ théologique? À eux de répondre sur le sort qu'ils lui font actuellement. Peut-être n'a-t-il, sous le soleil du post-postmodernisme, sa petite part de "France " qu'en catéchèse, discipline forcément à base de conviction personnelle? Sur cette situation, ce n'est pas Dieu qu'il faut interroger mais nous-mêmes.

\section{Conclusion - À propos de Dieu}

Que Dieu soit de moins en moins bien logé même dans les sciences consacrées à sa connaissance correspond à une trajectoire millénaire de sécularisation progressive. La Bible l'a inaugurée en désacralisant le cosmos au profit d'un Dieu unique. Jésus l'a poursuivie en luttant contre la pureté rituelle et en "désacrificialisant " et en "désacerdotalisant " la relation à son Père, action que nous nous sommes empressés de minimiser dans les institutions ecclésiales qui devaient prolonger son passage. Différents types de rationalité sont successivement apparus et ont pris la relève du mythe : la philosophique, la théologique, la scientifique, la technologique puis l'administrative. Les noms de Dieu ont perduré dans ces autres pans de la vie humaine que sont l'activité symbolique, sémiotique, langa gière, communicationnelle. La lancinante question de la transcendance ne s'y est jamais complètement tue, à la fois pour exprimer sa quête inquiète, ses succédanés ou son manque.

Devant les impasses de leurs perplexités, les chrétiens ont souvent eu recours à la notion de mystère. Le mystérieux, nous en fai sons l'expérience dans les moments qui, curieusement, participent de nos évidences les plus flagrantes : la vie, la mort, l'amour, le mal. Par contre, sa chosification en mystère-s bâillonne la réflexion et l'élan de la connaissance. Elle éloigne plutôt que d'inviter à progresser vers plus grand que soi.

Que souhaiter de mieux au Dieu de l'exégèse qu'il cesse d'être un mystère, une question qui demande une réponse, un problème qui implique sa solution, car, dans ces trois définitions ou descriptions le chercheur le circonscrit et l'éteint! Qu'il demeure une interrogation qui intrigue, un souci qui passe et revient, une attente, une mou- 
vance. Et que les exégètes aient le courage de la lucidité devant la nouvelle image qui apparaîtra à travers les formes bibliques fouillées par des yeux du vingtième siècle. "L'oracle ne me vassalise plus. J'entre : j'éprouve ou non la grâce ${ }^{6}$, "

6 René CHAR, extrait du poème «Calendrier », dans Fureur et mystère. Paris, Gallimard (nrf), 1962, p. 26. 